\title{
Collaborative reconstruction process design before an earthquake through the development of a pre-disaster planning program: a case study in Shinjuku Tokyo
}

\author{
Y. Kawasoe \\ Oriental Consultants Global Co., Ltd., Japan
}

\begin{abstract}
This study examines the development of pre-disaster planning program which shows the scenario to overcome the issues of reconstruction and revitalization after an earthquake. A provisional program has been applied based on the experience gained from the reconstruction of the Great Hanshin earthquake and the disaster prevention WS in Shinjuku Tokyo. The analysis made clear the followings three points. First, discussions about rebuilding and creation open spaces made the participants more enthusiastic about their future lifestyle comparing to discussions about road construction planning. Second, more participants were able to recognize their role in the reconstruction process through discussion with other participants about their roles in a future reconstruction. Third, the reconstruction manual based on the results of the WS contributed to clarify the common interest point and fascinate people about discussion on reconstruction scenario. The study points out that pre-disaster planning program contribute in making people become a part of the reconstruction scenario planning and motivate them to prepare for a future disaster.

Keywords: pre-disaster planning program, collaborative reconstruction, process design, method of participation, resilience.
\end{abstract}

\section{Introduction}

In general trend of disaster mitigation study, the concept of "resilience" is getting more important as well as it of "vulnerability" [1]. Seville et al. [2] defined that 
resilience is an umbrella concept not only survive but to be able to thrive through times of adversity. Aldrich [3] defined the community resilience as collaborative ability to overcome the stress and recover to the usual life after the disaster. Both of above definition include the capacity of all stakeholders in the area against the disaster. Chang-Richards et al. [4] show the figure to understand the resilience strength and weaknesses, and its indicators consist of "Leadership and Culture", "Network", and "Change Ready". From above, we can say that building resilience equals raising the capacity of those people itself and its networks, and be ready for future change. She also suggests the organisational resilience is created through the reconstruction process. This means not only hard infrastructure built after the disaster but also collaborative reconstruction can decrease the future risk by building resilience. We can hypothesis that experience of simulated reconstruction process could also build resilience beforehand, though the disaster risk reduction works basically target just after the hazard such as training for developing knowledge of hazard and evacuation.

The government of Tokyo prefecture enacted the program called "Pre-disaster reconstruction manual", and it is based on the community resilience by reconstruction residential unions [5]. However, the methodology of creating community resilience is obviously differs in every union and every distinct, and it has not been experimented yet. Draft post-2015 framework for disaster risk reduction from UNISDR [6] also suggest the necessity of visibility to implementation and place emphasis on stakeholders and their roles. Ichiko [7] researches the reconstruction experience drills in Tokyo which follows the manual and tries to grow community resilience through the drill, and conclude the necessity of program and manual in each distinct. On the other hand, the reconstruction program which is based on collaborative and comprehensive view and works is gathering more attentions through the experience of the reconstruction from the Great East Japan Earthquake. For example, Kobayashi [8] suggests the necessity of strategic program which plays a role of framework for the collaboration of various stakeholders. Also, Satoh [9] stated that the comprehensive recovery and reconstruction requires the mixture of top-down and bottom-up approaches, and also the methodology to create its mixture.

From the above, this study focuses on creating the methodology to design collaborative reconstruction process for building regional resilience through the practical works before earthquake happens. The collaborative reconstruction process design is consist of following three components. First is to share and coauthoring of future vision of the distinct achieved through its reconstruction. Second is to share and co-authoring of the process to the future vision. Third is to create the consciousness of role in collaborative works which accomplish the above two points.

\section{Methodology}

\subsection{Structure of study}

The next section analyses the reconstruction process of the Great Hanshin Earthquake, and figure out the scenario of collaborative reconstruction in 
regional scale. The framework of analysis is based on the Pre-disaster Reconstruction Manual of Tokyo (Figure 1). In the fourth section, the scenario is adapted to the workshop (hereinafter WS) which participants are residents, and the government and the consultant engineer support. The discussion at the WS is examined from the view of "the vision of future lifestyle and areal image" and "precursor and the collaboration of stakeholders", and its result will be summarized as pre-disaster planning program of residential WS. In the same way, the scenario is adapted to the WS which includes multi-stakeholders such as residents, enterprises, NPO, students, women and so on. The discussion is analysed and the effect is determined by comparing with the result of residential WS. In the last section, the "original pre-disaster reconstruction manual" is created from pre-disaster planning program, and the effect of utilizing the program is examined.

\begin{tabular}{l}
\begin{tabular}{l|l|}
\hline A. Just After Disaster & To rescue as many lives as possible and refuge \\
\hline $\begin{array}{l}\text { B. Refuge Life Term } \\
\text { C. Preparation Term }\end{array}$ & To assure life in refuges and houses during the period \\
\hline $\begin{array}{l}\text { D. Temporary } \\
\text { Reconstructioon Term }\end{array}$ & To enhance the life in temporary living and prevent secondary disasters \\
\hline $\begin{array}{l}\text { E. Reconstruction Term } \\
\text { Legend: }\end{array}$ & To advance temporary reconstruction for the successive approach to reconstruction \\
\hline
\end{tabular} \\
\hline
\end{tabular}

Figure 1: Framework of scene based on the Reconstruction Manual of Tokyo.

\subsection{Method of qualitative analysis}

This study use the qualitative analysis method suggested by Sato [10] as shown in Figure 2 in order to evaluate the phenomenon, which could not be evaluate from quantitative research, such as network among stakeholders. Firstly, the comments of people are recorded in WS. Secondly, all comments are documented. The documents are segmented and the burden of argument, and are decontextualized and extracted as "code". At that time, all codes obtain its "speaker", "scene", "theme". The codes are categorized by their "scene" and "theme", and the relationships between the codes are classified to "cooperation: opinions based on a same issue", "derivation: a solution to an issue, or an opinion derived from another opinion", or "conflict: opinions with contradiction". The discussion of WSs are structured and analysed through this process.

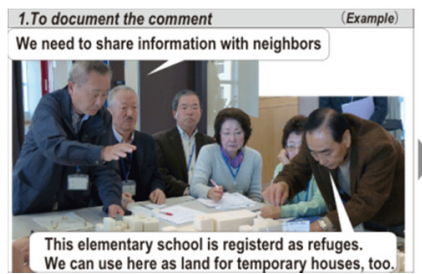

Figure 2: Method of qualitative analysis. 


\section{Reconstruction scenario from the Great Hanshin Earthquake}

This section reads the reconstruction scenario from the Great Hanshin Earthquake 1995 as the standard for creating the pre-disaster planning program in metropolitan area. Lots of study has focused on the reconstruction after the Great Hanshin Earthquake such as Satoh [11]. However its scenario is not described as a thesis, and a primary source is necessary to understand it. Therefore, the author uses the documentary movies. In a lot of movies, the one from Aoike [12] is chosen because the product include not only just after the earthquake but also reconstruction until 1999. The length of the movie is 14 hours and 38 minutes. The movies are qualitatively analysed with the methodology written above, then are chronologically ordered, and finally separated to the scene of Figure 1. The succession of those scenes is the reconstruction scenario.

The validated reconstruction scene is structured as Figure 3, which is the one of reconstruction preparation scene. By examining all scenes, 27 themes of works in 5 scenes are elucidated (Figure 4).

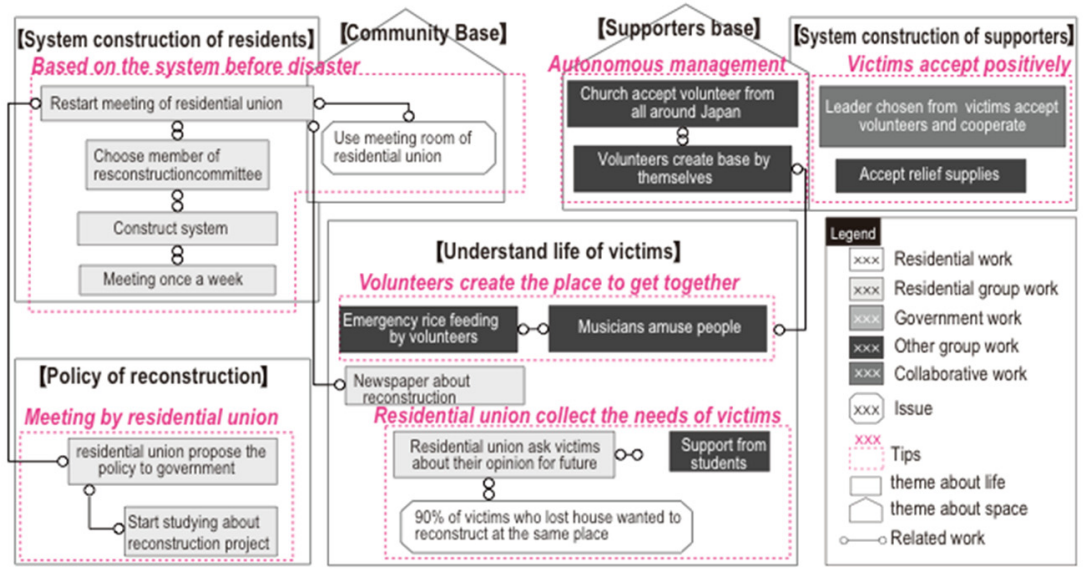

Figure 3: Qualitative analysis of reconstruction preparation scene.

\section{Case study of adapting the reconstruction scenario to residential WS for pre-disaster planning program}

Residential WS was held on 2 October 2014. Before the WS, the attendants understand the process of reconstruction through watching some part of the above movies, and also recognize the issue and characteristic of the area where they live by walking and making original maps. Figure 5 shows the framework of the residential WS, and how the reconstruction scenario is adapted. 27 themes of reconstruction scenario are written on the flag, and the attendants stick the flag to the model with considering their role and place. The model is updated as scene 


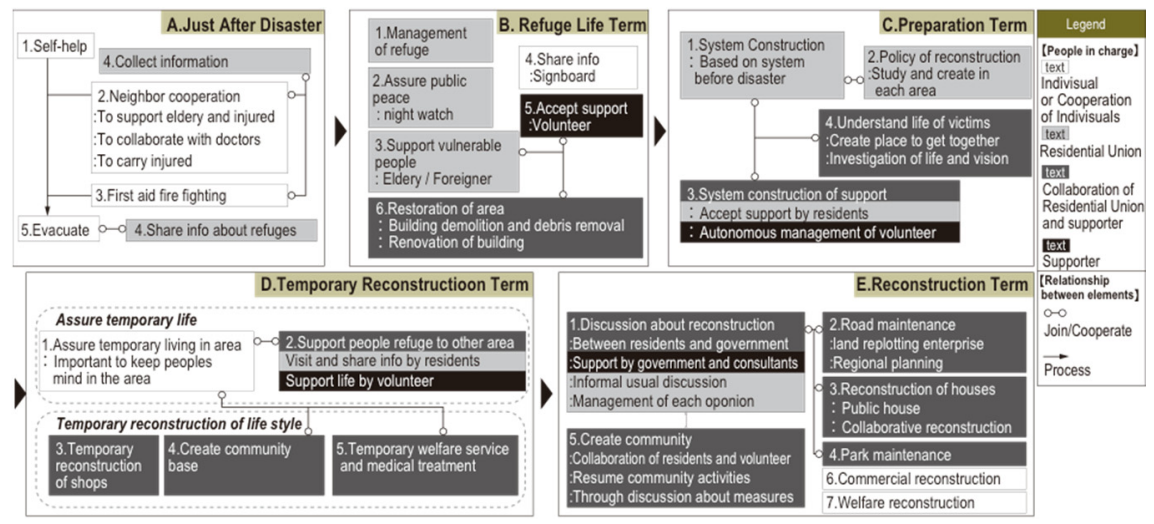

Figure 4: Reconstruction scenario from the Great Hanshin Earthquake.

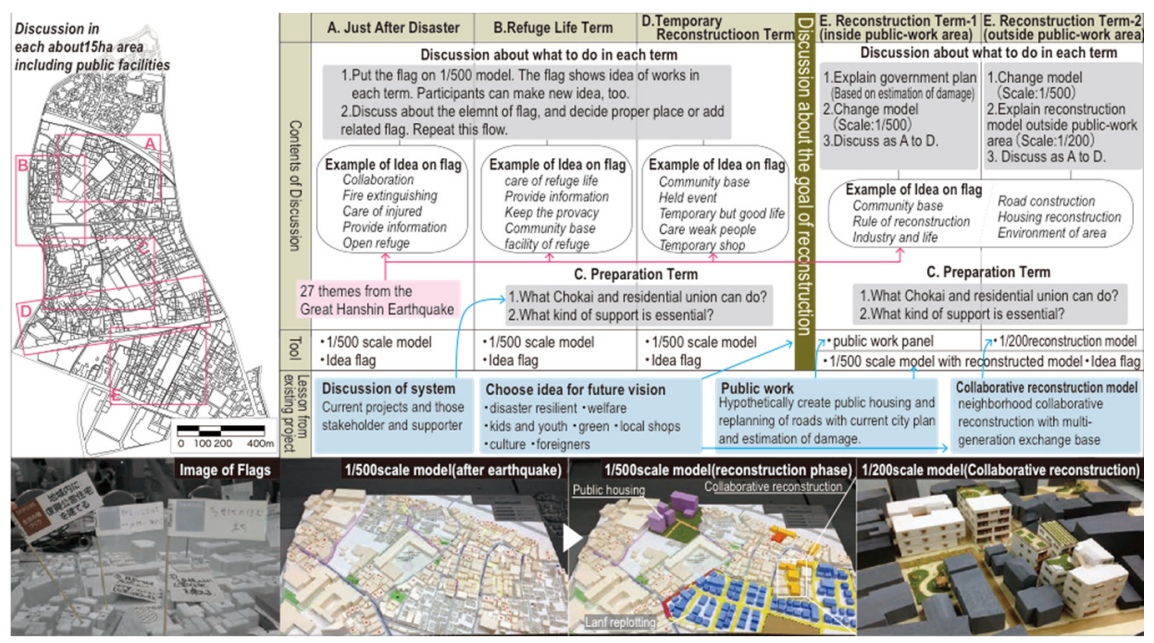

Figure 5: $\quad$ Framework of residential WS.

proceed, and the changed point and the element of flags are explained by the facilitator. The model of reconstruction scene is created based on the simulation of hazard and the vision of areal master plan which includes community, welfare, environment, multi-culture, and so on. The scale of model is around 15 ha and the attendants separated to 5 teams.

The result and the development of the discussions are structured as Figure 6, which is the reconstruction preparation scene of group E. Each opinion are categorised from the view of "future vision and lifestyle", and "stakeholder". The relationship between the opinions are categorised to "cooperation", "conflict", and "derivation". The examinations are as follows. 


\subsection{Exploration of residential reconstruction union system}

Currently, neighbourhood association called "Chokai" is the residential group which deals with issues in neighbourhood. However, some teams explore the reconstruction union system for reconstruction in order to solve problems after disaster and achieve vision. Group $\mathrm{A}$ and $\mathrm{C}$ compose of large Chokai, and it is determined that the current Chokai directory launch reconstruction union for reconstruction after the disaster. On the other hand, Group B and D consist of small Chokais, and the collaboration of Chokai for launching residential union is discussed. In the discussion of Group B, the area is based on the place to evacuate, and Group D uses the cooperation system for annual local festival. Group E considered integrating Chokai with store associations and cooperation association for reconstruction union in order to utilize the characteristic of the area.

\subsection{Limitation of discussion within the current system}

Regarding the process of discussions regarding residential reconstruction union, group A, D and E discusses alternative ideas such as renewal of association for reconstruction union because of the capacity shortage of current association. On the other hand, group $\mathrm{B}$ and $\mathrm{C}$ discuss reconstruction process only within current neighbourhood association, then they can understand the issue within current system, but they cannot make alternative ideas. From above, all groups discussed about the alternative system for reconstruction union, but concrete discussion cannot be enfolded without key person such as young people or foreigners living a while in Japan. Therefore, it is clear that discussion within current system has limitation.

\subsection{Idea includes future lifestyle from discussion about spatial improvement}

All teams have demands to future lifestyle with improving spatial condition in discussions. In those demand, the "Road improvement" is only connected with "Park" in team B. All of other demands are based on "Public houses", "Cooperative reconstruction", and "Park". Those have more opportunity to draw the demands of local residents than road "improvement".

Pre-disaster planning program structured through residential WS is realistic and based on the current situation, however it means the current system is not enough for achieving the ideal reconstruction for attendants. Regarding the ideal reconstruction vison, important issues are discussed and the projects achieving the vision are also discussed.

The contents of pre-disaster planning program structured through residential WS are shown on the left side of figure 7.

\section{Case study of adapting reconstruction scenario to multi-stakeholder WS for pre-disaster planning program}

The attendants to the multi-stakeholder WS include a representative of residents, Shinjuku government, NGO, architect, consultant, and so on. The WS was separated to 2 times, and 110 people from 61 groups attended. 
Before starting the WS, the characteristic of the area and the result of residential WS are explained to the attendants, because some of them are not familiar with the risk of area and the state of disaster prevention progress. The attendants are divided into 3 teams. The method of structuring the discussion is basically same as one on residential WS, however in order to examine from the view of "the role of each stakeholder and its network", each opinion is classified into "charge: the opinion to be in charge of something", "demand: the opinion to request other stakeholder to do something", and "issue: the opinion to raise a question about some issue". Also the relationships between the opinions are classified to "demand: the opinion to request other stakeholder to do something", "collaboration: collaborative action from some stakeholders", and "support: one stakeholder support another" in addition to "cooperation", "conflict", and "derivation." The upper side of Figure 6 shows the matrix based on "Speaker" and "theme". 27 per 32 parties recognize at least one role in reconstruction process, and 26 per 27 themes are discussed. The analysis of discussion is as follows.

\subsection{The process of recognizing own role developed through WS}

Based on Figure 6, the developments of discussions are classified into 6 groups from the view of "presence and clarity of party which is in charge of one activity for reconstruction". Table 1 on Figure 6 summarizes the classification.

1) Classification of discussions with parties in charge of discussed activities

Discussions with stakeholders in charge of discussed activities contains (1) the cooperation of stakeholders is recognized, (2) one or some stakeholders are recognized, but the cooperation is not recognized (3)-1 some recognize the role but not all. These discussions are discovered in 31 themes in 43 themes.

2) Classification of discussions without stakeholders in charge of discussed activities

Discussions without stakeholders in charge of discussed activities contains (3)-2 No one recognizes the role, though there is demand to specific stakeholders, (4) No on recognize the role, though there is demand to unspecific stakeholders,

(5) No demand and no recognition of role.

From the above, some recognition as having a role in the reconstruction process is common knowledge for the groups, but on the other hand, some recognition becomes evident through WS discussion such as cooperation, support and demand between stakeholders. The theme of (3),(4),(5), which the role is not recognize, have to be discussed more through finding the stakeholder based on the demand and cooperation.

\subsection{The feature of each stakeholder's remarks and its effect on the discussion}

The development of discussion creating recognition of one's role is analyzed from the view of feature of the speaker. 


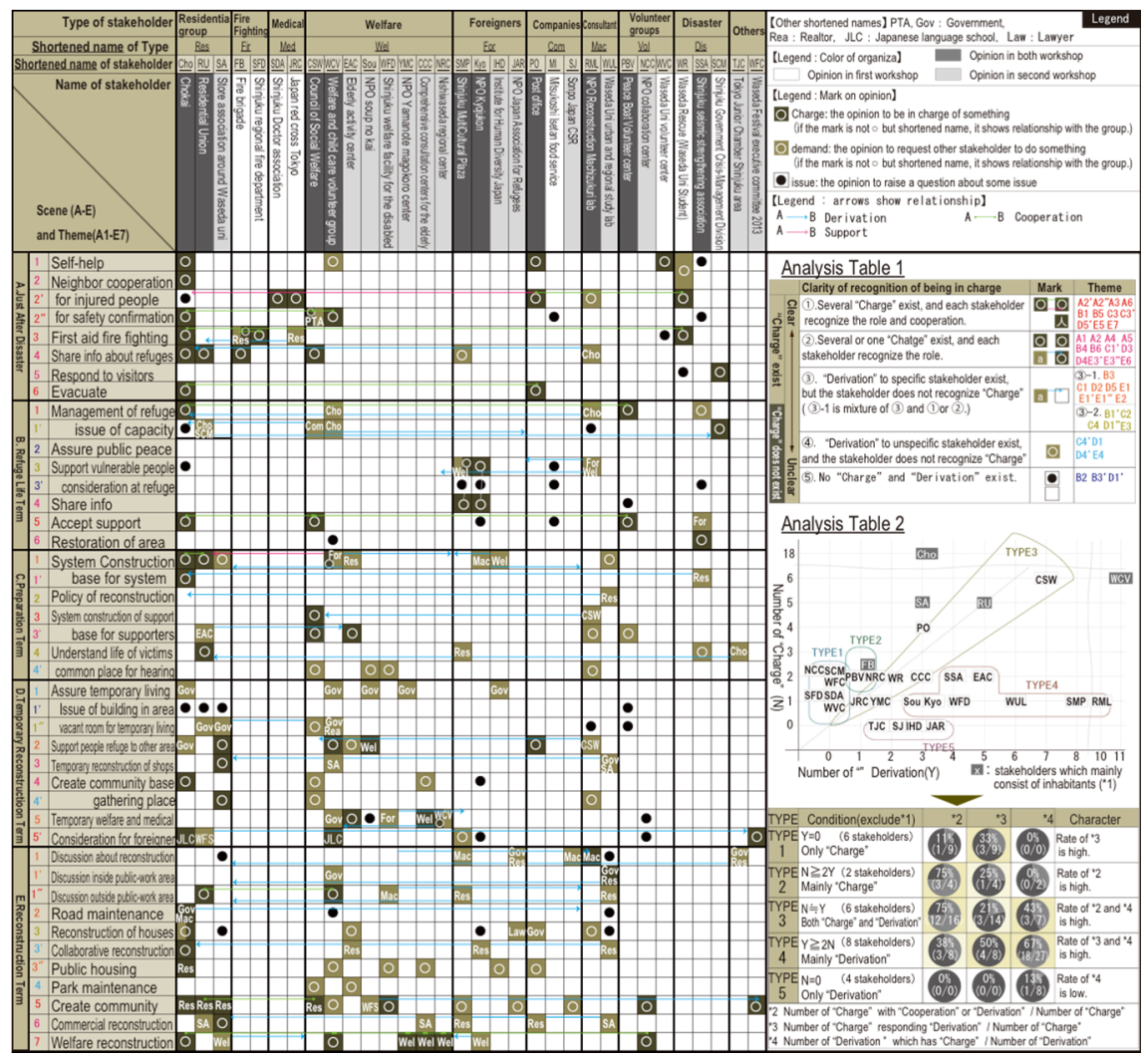

Figure 6: Analysis of a multi-stakeholder workshop.

1) The feature of remarks by stakeholders which mainly consist of inhabitants

Stakeholders which mainly consist of inhabitants have lots of remarks, and the recognition of their own role is seen frequently. The fire brigade has recognition in the role of first-aid firefighting. The district welfare commissioner has recognition as in charge of welfare in addition to similar role as Chokai and residential union, and it has large number of demands.

2) The feature of remarks by stakeholders which most members are not residents The stakeholders which most members are not residents but commuting from other areas are classified into 4 types from the view of "number of remarks as recognizing the role" and "number of remarks of demand" (Table 2 on Figure 6). a) The effect of the stakeholders which remarks are either recognizing role or demand

TYPE 1 includes the stakeholders which remarks are only recognizing the role, and those remarks are responding to the demand from other groups. The cooperation with other stakeholders is relatively few. TYPE 5 includes the stakeholders which remarks are only demand to others stakeholders, and those demands are relatively less responded. These two types have comparatively less influence on other groups. 
b) Arousing the recognition of own role for all attendants by the remarks from stakeholders who comment both demand and own role.

TYPE 2 is the groups which remarks are mainly recognition of own role $(\mathrm{N} \geqq 2 \mathrm{Y})$, and it has relatively high rate about cooperative works. TYPE 4 is the groups, which remarks are mainly demands to other groups $(\mathrm{Y} \geqq 2 \mathrm{~N})$, and accepted rate of demand is relatively high. TYPE 3 is the groups, which remarks about recognition of role and remarks about demands are almost same amount, has relatively high rate about both of cooperative works, and accepted rate of demand. From above, the groups which remarks are consist of both demands and recognitions of roles have more connections with other groups. The recognition of roles and network among groups are clarified through repeat of those demands and acceptance.

Though it is up to the groups if main remarks are demands or recognition of roles, groups can contribute to create effective discussion which clarifies each group role and network of groups. The pre-disaster planning program structured through multi-stakeholder WS is shown on the right side of figure 7. Comparing to the program structured through residential WS, more issues of reconstruction are responded by groups. The recognition of role of un-residential stakeholders clarifies the role and network of residential groups as well.

\section{Reconstruction manual based on the program}

Questionnaires regarding valuation of WS and wishes for utilizing the predisaster planning program are prepared after both residential and multistakeholder WSs. 51 out of 68 people $(75 \%)$ of residential WS and 27 out of 62 (44\%) answered the questionnaire.

\subsection{Valuation of WS}

Valuation of WS is shown on left side of Figure 8. Approximately $90 \%$ answerer of residential WS could think of future vision after disaster. All residents and more than $90 \%$ un-residential answerer of multi-stakeholder WS could understand the process of reconstruction. Approximately $90 \%$ of both residential and un-residential groups could understand the character and thought each other.

\subsection{Wishes to utilize the pre-disaster planning program as reconstruction manual}

More than 90\% answerer of both residential and multi-stakeholder WS thought necessity of making reconstruction manual based on the result of WS.(right side of Figure 8) Regarding the contents of the manual, "cooperative network system among residential group and other groups" and "Activities by residents after disaster" is most essential. "Cooperative system among multi-stakeholders" and "Supposed issues of every scene" are also necessary. From above, attendants of WS wish for the reconstruction manual which shows the activities by multistakeholders based on the works of residents for issues of every scene after the disaster. 

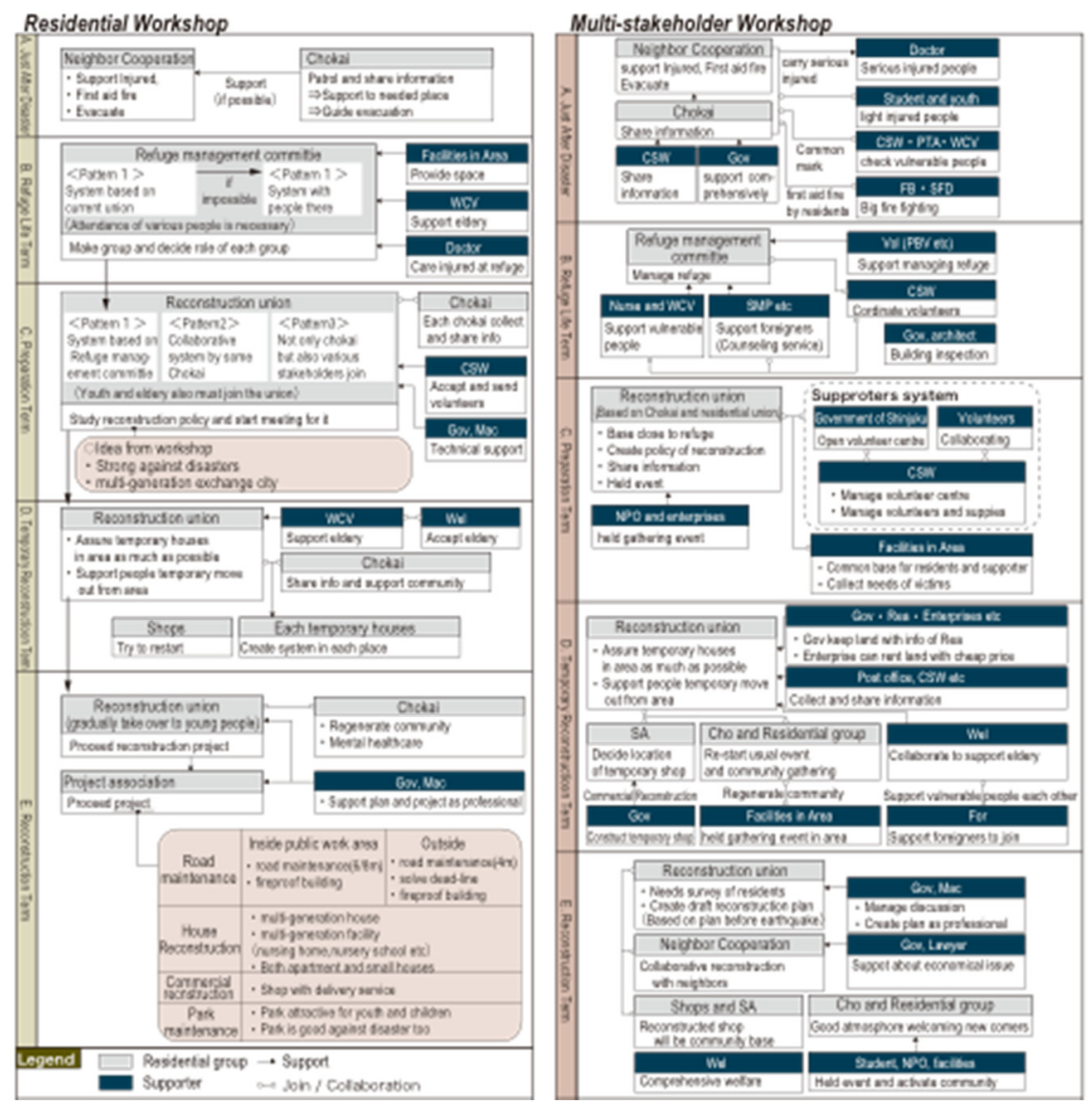

Figure 7: Contents of pre-disaster planning program by residential WS (left) and multi-stakeholder WS (right).
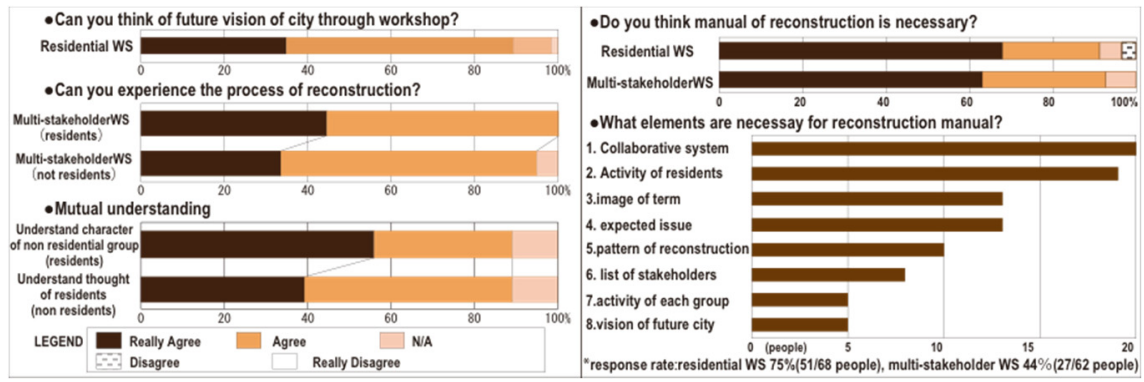

Figure 8: Result of interview. 


\subsection{Effect of pre-disaster manual based on the program}

The pre-disaster reconstruction manual is created and published to residents and other attended groups. In order to validate the effect of the manual, interview was held to 19 groups which attended either residential or multi-stakeholder WS.

Discussions on the interview was classified into "recognition of own role" and "demand to other groups" as done in the previous section. As a result, 42 times of "recognition of own role" remarks and 56 "demand to other groups" remarks are defined. By analyzing defined remarks, following two effects are confirmed.

1) Awareness of theme which attracts lots of interest

Scatter plot about amount of "recognition of own role" remarks and "demand to other groups" remarks on every theme is shown in Figure 9. Issues are concentrated at a few themes. Because the interview was held group by group, the themes attract many interest are common issue. It would be differs area by area, and by stakeholders of the area. The theme should be discussed more to update the manual to meet the condition of area.

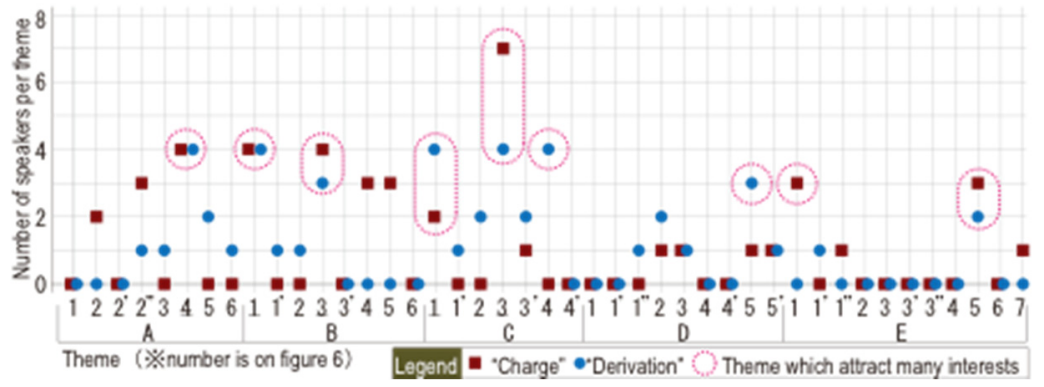

Figure 9: $\quad$ Scatter plot of remarks and typical opinions.

2) Facilitation of attendants to the discussion of pre-disaster planning

TYPE1 and TYPE2 had either "recognition of own role" or "demand to other groups" remarks on the WS, but 2 out of 3 TYPE1 groups and 1 out of 2 TYPE2 groups remarked both remarks through the interview. Also, all groups, which had nether remarks on the WS, have both or either remarks through the interview. Overall, presenting pre-disaster manual increases the awareness of their roles and demands to other groups.

\section{Discussions}

This study analyzes the process of structuring the pre-disaster planning program, and following points are verified.

- Structuring pre-disaster planning program through residential and multistakeholder WS brings about the understanding future vision, and consciousness of process and collaborate network system to achieve it: This achieves both. 
- Utilizing pre-disaster planning program as reconstruction manual contributes to clarify the issue of the area and to promote the consciousness of collaboration. In conclusion, "Creating pre-disaster planning program" contributes in making people become a part of the reconstruction scenario planning, and "reconstruction manual based on the program" can be the common indicator to advance preparation beforehand. These effects equal to strengthen community resilience from the views of "Networks" and "Change Ready". Continuous effort to find proper leader and to build the resilient culture with using the reconstruction manual will be necessary. It will be combined idea of resilient city and Machizukuri idea in Japan, and this would be the concept idea to continue developing my research. As an outcome of the study, figure10 shows the methodology to design collaborative reconstruction scenario through the development of a pre-disaster planning program.

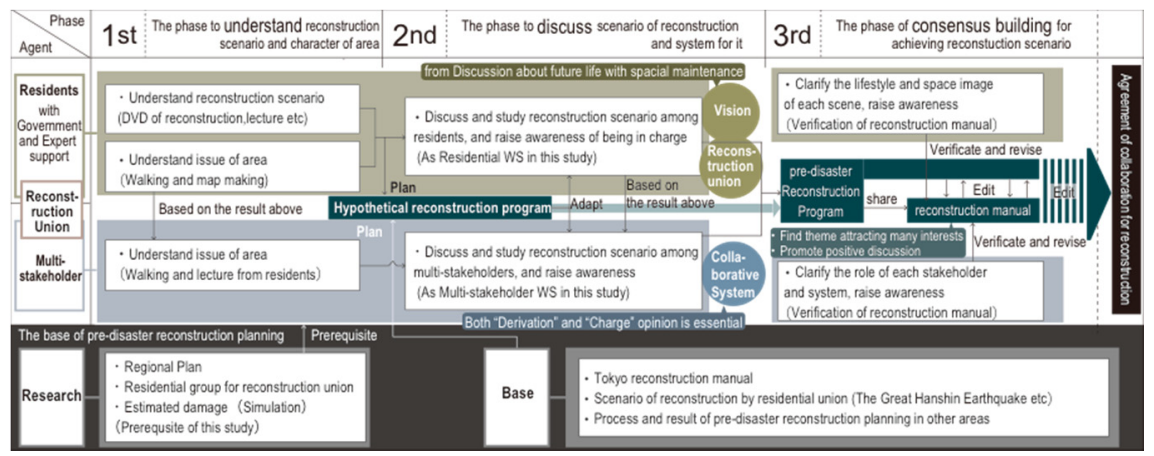

Figure 10: Methodology to design collaborative reconstruction scenario.

\section{References}

[1] Oyane, Urano, Tanaka, Yoshii, Saigai-syakaigaku-nyumon (Disaster socialism) 2007.

[2] Seville E., Brunsdon D., Dantas A., Le Masurier J., Wilkinson S., Vargo J. Organisational resilience: Researching the reality of New Zealand organisations, Journal of Business Continuity \& Emergency Planning, 2(3), pp. 258-266, 2008.

[3] Aldrich D. P., The Power of People, Social Capital's Role in Recovery from the 1995 Kobe Earthquake: Natural Hazards, 56(3), pp. 561-595, 2012.

[4] Chang-Richards A., Vargo J., Seville E. Organisational Resilience to Natural Disasters: New Zealand's Experience, China Policy Review, Vol. 10, pp. 117-119, 2013.

[5] Tokyo Reconstruction Manual, 2003.

[6] Post-2015 framework for disaster risk reduction zero draft submitted by the co-chairs of the preparatory committee, United Nation, http://www.wcdrr.org/preparatory/post2015 
[7] Ichiko T, Pre-disaster planning for post-disaster recovery during 2000's in Nerima Ward Tokyo, Tokyo Metropolitan University Journal of Urban Science, Vol.3, pp. 103-114, 2009.

[8] Kobayashi H, Redesigning Planning through Experience of Reconstruction Planning and Disaster Prevention Planning, Urban Planning 61(5), pp. 3741, 2012.

[9] Satoh S, The Framework of Reconstruction Machizukuri: The Vision and Issues from some Experimentation of Infrastructure Planning, Machizukuri Vol.34, 2012.

[10] Satoh I, Sitsuteki data bunsekihou genri houhou jissen (Qualitative analysis methodology its principle, method and practice), 2008.

[11] Satoh S, Fukkoumachizukuri no jidai (Era of reconstruction planning), 2006.

[12] Aoike K, Kiokunotameno-rensaku Nodahokubu Takatorino hitobito 1-14 (Documentary of Nodahokubu Takatori reconstruction vol.1-14), 1999. 